\title{
Experiencia en la reconstrucción de ligamento cruzado anterior sin desinserción de isquiotibiales con túnel femoral anatómico fuera-dentro
}

\author{
Experience in anterior cruciate ligament reconstruction using hamstring autograft \\ with preserved insertion and anatomical femoral tunnel outside-in drilling
}

José Noé Salazar López, *, Alberto Hernán Rodríguez Pesina, * Luis Guillermo Yáñez Mejía, * Juan Manuel Elizalde Peña, ${ }^{*}$ Álvaro Vázquez-Vela Echeverría, ${ }^{*}$ Fabián Marín Yee, ${ }^{*}$ Otilia Gallegos De la Torre*

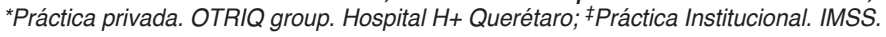

\begin{abstract}
Resumen
A pesar de las controversias que existen con las diferentes técnicas de reconstrucción del ligamento cruzado anterior, creemos que una buena opción es la plastia ligamentaria con autoinjerto grácil-semitendinoso sin desinserción tibial, túnel femoral anatómico fuera-dentro y fijación femoral anterógrada con tornillo interferencial. Esto debido a que el autoinjerto sin desinserción preserva sus propiedades biológicas, mejorando la propiocepción. Además de que la técnica túnel fuera-dentro con la guía femoral de cruzado posterior permite una perforación más horizontal, logrando un mayor ángulo de doblamiento del injerto Graft bending angle que mejora la estabilidad anteroposterior-rotacional y reduce el estrés de la plastia. Por último, para la fijación preferimos usar tornillos interferenciales, puesto que es el sistema que sigue vigente a pesar de los diferentes métodos y del paso del tiempo. Sin mencionar que se evitan problemas de fijación tibial, ya que no se realizó desinserción tibial del autoinjerto. Compartimos nuestra experiencia con 32 procedimientos.
\end{abstract}

Palabras clave: Ligamento cruzado anterior, autoinjerto isquiotibial, túnel femoral anatómico, ángulo de doblamiento del injerto, tornillo interferencial.

\begin{abstract}
Despite of the controversies with the different techniques for anterior cruciate ligament reconstruction, a good option is a surgery using hamstring autograft with tibial preserved insertion, anatomical femoral tunnel outside-inside drilling and femoral fixation with an extraarticular interference screw. Because a graft without disinsertion preserves its biological properties, improving proprioception. In addition, the outside-in drilling technique allows to make an horizontal tunnel, achieving a greater graft bending angle that improves anteroposteriorrotational stability and reduces stress on the plasty. Finally, we prefer to use interference screws, considering that it is a reliable system that survive the passage of time and is the only system that allows to keep graft insertion. We share our experience with 32 procedures.
\end{abstract}

Keywords: Anterior cruciate ligament, hamstring autograft, anatomical femoral tunnel, graft bending angle, Interference screw

\section{Introducción}

La reconstrucción de ligamento cruzado anterior (LCA) es uno de los procedimientos más comunes a nivel mundial, estimándose que tan sólo en EUA se realizan 200,000 procedimientos al año. ${ }^{1}$ La complejidad y evolución de esta ciru-

Correspondencia:

José Noé Salazar López

E-mail: nsalazarl.ot16@gmail.com

Recibido: 26-04-2021. Aceptado: 09-05-2021. gía causa gran controversia en el tipo de injerto a utilizar en la técnica para realizar los túneles y en el sistema de fijación. ${ }^{2-4}$ Una opción a este procedimiento es la plastia con autoinjerto grácilsemitendinoso ipsilateral sin desinserción tibial (T4) (Figura 1), perforación del túnel femoral anatómico fuera-dentro (TFAFD) con la guía femoral

Citar como: Salazar LJN, Rodríguez PAH, Yáñez MLG, Elizalde PJM, Vázquez-Vela EÁ, Marín YF, et al. Experiencia en la reconstrucción de ligamento cruzado anterior sin desinserción de isquiotibiales con túnel femoral anatómico fuera-dentro. Orthotips. 2021; 17 (3): 138-142. https://dx.doi.org/10.35366/100622 

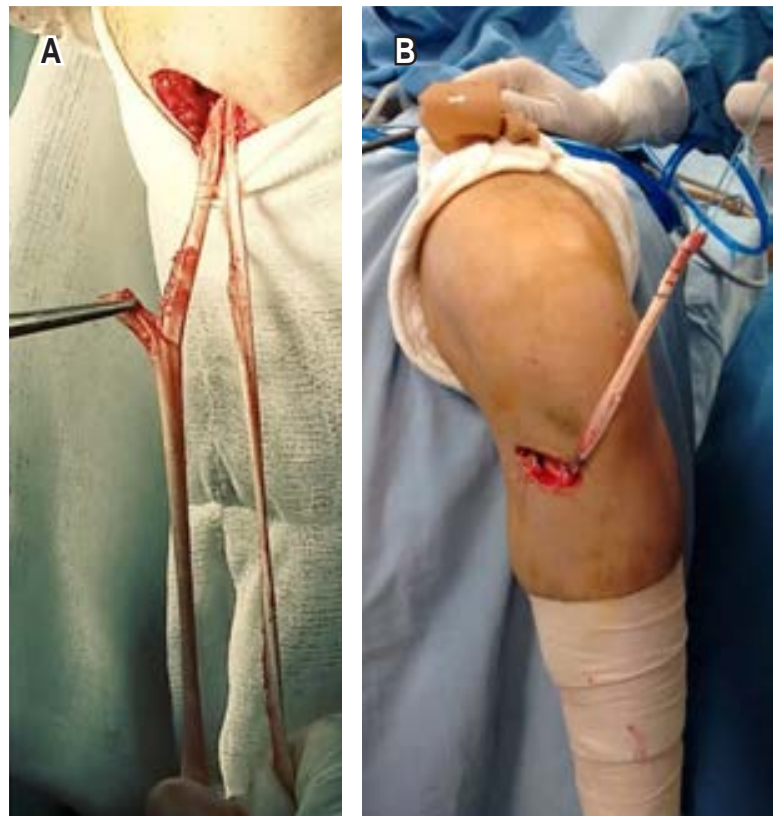

Figura 1: Autoinjerto grácil y semitendinoso. A) Gran inserción facial. B) Injerto T4.

de cruzado posterior y fijación femoral con tornillo interferencial anterógrado $(\mathrm{TI})$. Este procedimiento ofrece las siguientes ventajas: 1) al no desinsertar de la tibia el autoinjerto grácil-semitendinoso se preserva el aporte vascular y la inervación nativa, lo que puede mejorar la propiocepción;5,6 2) la perforación femoral con técnica fuera-dentro utilizando la guía femoral de cruzado posterior permite realizar un túnel anatómico, esto debido a que la visualización directa a través del portal medial permite identificar las referencias óseas, la cresta del residente o intercondílea lateral (Figura 2) y la cresta bifurcada. ${ }^{7,8}$ Es importante recordar que un túnel anatómico, es decir, una perforación más horizontal aumenta el ángulo de doblamiento del injerto Graft bending angle, lo que mejora la mecánica articular (Figura 3), ya que se reduce el estrés del injerto y aumenta no sólo la estabilidad anteroposterior, sino también la estabilidad rotacional; ${ }^{9}$ 3) para la fijación preferimos usar tornillo interferencial femoral anterógrado, puesto que este sistema logra la fuerza requerida para realizar las actividades de la vida diaria (454 Newtons), además de ofrecer una rigidez adecuada, por eso se sigue considerando el estándar para la fijación; ${ }^{10}$ 4) por último, mencionamos otra ventaja de la no desinserción del autoinjerto, pues se está evitando el riesgo de falla por deslizamiento secundario a la pérdida de la fijación, riesgo que se incrementa por la menor densidad mineral ósea en tibia. ${ }^{11}$

El protocolo postquirúrgico utilizado es: de 0 a dos semanas con protocolo POLICE, sin apoyo, movilidad $0-90^{\circ}$ y rodillera en extensión al dormir; de dos a seis semanas con apoyo total, movilidad libre y bicicleta fija; de seis a 12 semanas con movilidad completa, fortalecimiento y ejercicio aeróbico; 12-24 semanas carrera en línea recta, ejercicios de coordinación y agilidad, y después de la semana 24 , cambios de dirección y regreso a las actividades deportivas.

El objetivo es compartir nuestros resultados con esta opción quirúrgica, ya que consideramos que los resultados pueden ser buenos y reproducibles.

\section{Material y métodos}

Estudio retrospectivo, observacional y analítico. Nivel de evidencia 3.

Se evaluó la funcionalidad postquirúrgica en pacientes sometidos a reconstrucción de LCA con
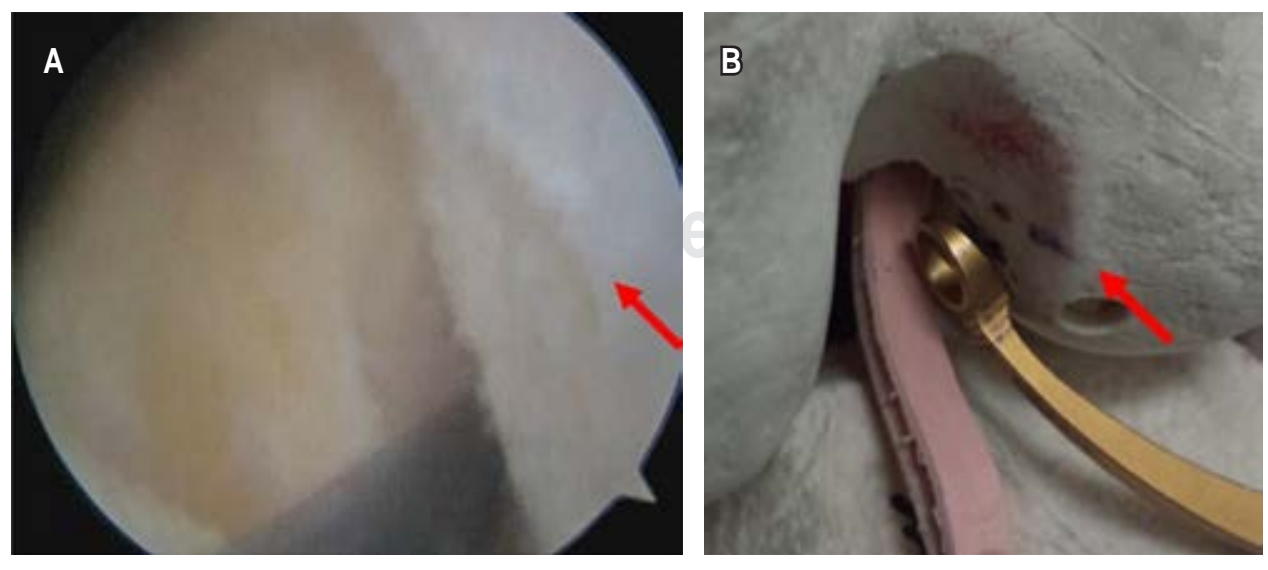

Figura 2:

Cresta del residente 0 intercondílea lateral. A) Foto artroscópica. B) Modelo anatómico. 


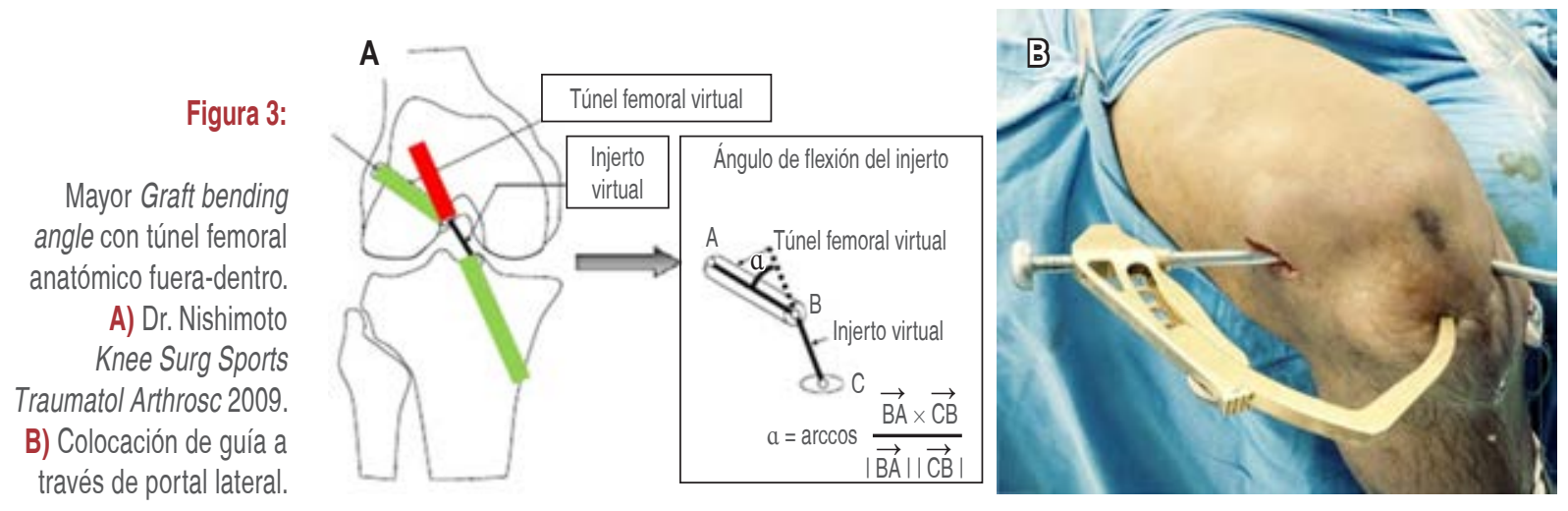

injerto, túnel femoral y fijación previamente mencionados de nuestra práctica privada e institucional en un periodo de abril de 2015 a noviembre de 2018 en la ciudad de Querétaro y San Luis Potosí con los siguientes criterios de selección: a) inclusión: paciente con lesión de LCA entre 16-50 años sin deformidad varo-valgo o deformidad Krackow I, b) exclusión: paciente con lesión de LCA y lesión condral Outerbridge III-IV, c) eliminación: paciente con lesión de LCA > 18 meses, lesión multiligamentaria, amputación de ambos isquiotibiales, deformidad varo-valgo Krackow > II o cirugía previa en rodilla.

Se realizó la evaluación Tegner Lysholm Knee Scoring Scale a las ocho semanas; clasificando el resultado como: excelente con puntaje $>90$, bueno entre $84-90$, limitado entre $65-83$ y mal puntaje < 65. Utilizamos la evaluación del sitio www. orthopaedicscore.com.

Consideraciones éticas. Investigación con riesgo menor que el mínimo (Declaración de Helsinki), ya que la posibilidad de causar alteración física o psicológica en el sujeto es prácticamente nula. Esto debido a que no se pretende realizar cambio alguno a la técnica quirúrgica, ni se evaluará resultado con nuevo injerto, instrumental o sistema de fijación.

\section{Resultados}

Durante el periodo previamente mencionado, 32 plastias cumplieron con los criterios de inclusión, mientras que se excluyeron y eliminaron, cinco y 10 , respectivamente. De estos 10 pacientes, tres presentaban lesión crónica, dos lesión multiligamentaria, dos deformidad en varo, dos artroscopia previa y una amputación de ambos isquiotibiales.

Dentro de los incidentes y hallazgos quirúrgicos de nuestros pacientes queremos mencionar que en cuatro casos ocurrió amputación de músculo grácil, por lo que la reconstrucción fue con tres bandas (T3) con un diámetro final de injerto de $7 \mathrm{~mm}$, en tanto que en un caso el autoinjerto midió $6 \mathrm{~mm}$ a pesar de no tener problema con la toma del mismo. También logramos realizar la plastia sin eliminar el remanente de LCA en 12 casos (técnica augmentation), tres de los cuales fueron en plastias con IT3.

Los puntajes fueron desde 46 hasta 96; sin embargo, afortunadamente se observaron buenosexcelentes resultados en $93 \%$ de las plastias (excelentes resultados en 18 pacientes y 12 reconstrucciones con buenos resultados). De estos pacientes el promedio con excelentes resultados fue de 92.8 puntos, mientras que para los pacientes con buen resultado el promedio fue un puntaje de 88 . También se observó resultado limitado en un paciente y resultado malo en una reconstrucción (Tabla 1). El paciente con resultado limitado tuvo un total de 83 puntos, esto debido a referir leve cojera, dolor durante actividad intensa y chasquido sin bloqueo articular, lo que no limitaba sus actividades de la vida diaria ni sus actividades laborales. En el caso

Tabla 1: Resultado de la evaluación a las ocho semanas.

Evaluación Tegner Lysholm Knee Scoring Scale

\begin{tabular}{lcc}
\hline Clasificación & $\mathrm{n}(\%)$ & Media-puntos \\
\hline Excelente & $18^{*}(56.0)$ & 92.8 \\
Bueno & $12(37.5)$ & 88.0 \\
Limitado & $1(3.0)$ & 83.0 \\
Malo & $1(3.0)$ & 46.0 \\
Total & $32(100.0)$ & \\
\hline
\end{tabular}

${ }^{*}$ Incluye T3 y Augmentation. 


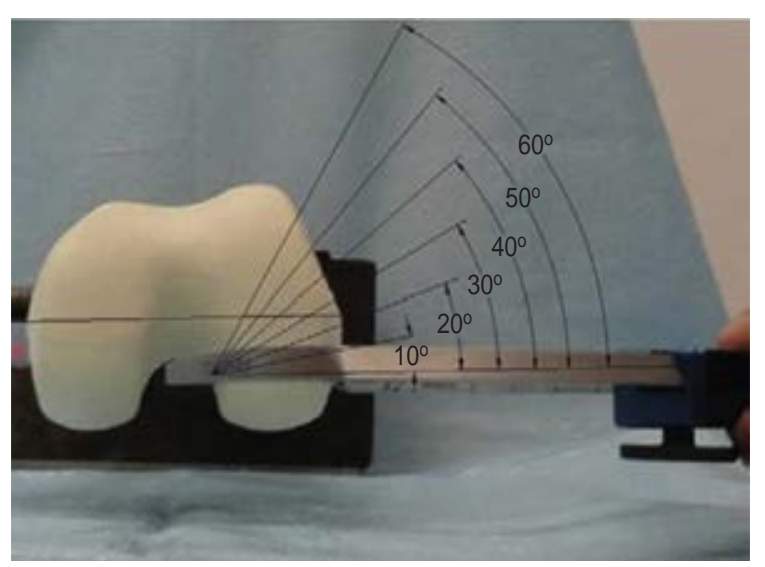

de nuestro paciente con malos resultados (46 puntos), éste refirió realizar una carrera de $10 \mathrm{~km}$ a las cuatro semanas de operado.

En todas las reconstrucciones con T3 y con técnica Augmentation se logró un puntaje promedio de 92 puntos (resultados excelentes).

\section{Discusión}

A pesar de lo frecuente que resulta la lesión de este ligamento, hoy en día algunos detalles de la técnica quirúrgica siguen en discusión. Sin embargo, desde hace aproximadamente 10 años era evidente la inestabilidad rotacional residual en $10-20 \%$ de los pacientes sometidos a la reconstrucción, para más tarde confirmar que una causa común de falla es un túnel femoral no anatómico. ${ }^{12}$ Ésta es la razón por la que actualmente es preferible una perforación anatómica del túnel femoral. A continuación se comparten algunos detalles importantes con esta técnica quirúrgica.

Al intentar mejorar las condiciones biológicas del injerto al no desinsertar en la tibia es necesario el uso de un tenótomo abierto, lo que incrementa el riesgo de amputación durante la toma del autoinjerto grácilsemitendinoso si no se ha realizado una adecuada liberación de las inserciones faciales. Una vez tomados los injertos isquiotibiales, se limpian y pliegan, dejando una sutura en el extremo libre para la tracción y colocación del mismo, lo que ahora estaría formando el autoinjerto conocido como T4 (Figura 1). Antes de efectuar la perforación femoral, la guía femoral se calibra a $60^{\circ}$; el lente artroscópico se cambia al portal medial, lo que permite identificar directamente la cresta del residente para introducir la guía femoral a través del portal lateral y orientar la misma a $20^{\circ}$ a partir del eje transepicondíleo (Figura 4$).{ }^{8}$ La per-

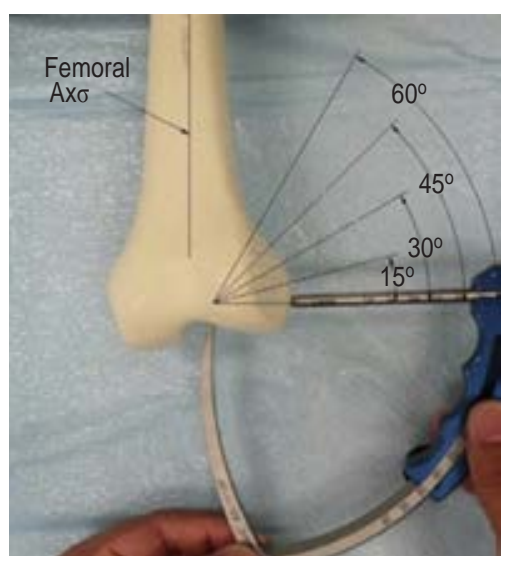

Figura 4:

Orientación de guía para perforación femoral anatómica fuera-dentro. Tomado de: Lubowitz JH, et al. ${ }^{8}$ foración tibial se realiza de forma habitual. Una vez perforados ambos túneles, con ayuda de pinza de cuerpos libres se introduce la sutura que traccionará el injerto a través del túnel tibial y posteriormente se recuperará la misma por el túnel femoral de forma retrógrada. Antes de la fijación, estrictamente hablando, no sería necesaria la colocación del tornillo interferencial en tibia, ya que ese extremo continúa fijo, pero se recomienda colocarlo para reducir la probable formación de algún quiste pretibial. Así que ahora la tensión y fijación del injerto dependen de la colocación del tornillo interferencial en el lado femoral, para lo cual se sugiere realizar una incisión que permita la correcta visión del túnel y así evitar problemas con la colocación del tornillo.

Actualmente, a pesar de contar con varios y muy buenos sistemas de fijación, preferimos usar el tornillo interferencial, puesto que es un sistema confiable que ha sobrevivido al paso del tiempo y es el único que permite conservar la inserción del injerto en tibia.

\section{Conclusiones}

Sugerimos una estricta liberación de tejidos blandos antes de intentar la toma del autoinjerto, ya que al utilizar un tenótomo abierto la posibilidad de amputar el injerto se incrementa.

Pensamos que probablemente los buenosexcelentes resultados observados con esta técnica se relacionan con la eliminación de esos puntos débiles de la plastia de LCA. Es decir: 1) al no desinsertar el autoinjerto se preserva el aporte vascular y la inervación nativa, además de eliminar el riesgo de pérdida de fijación por menor densidad mineral ósea tibial, 2) al realizar un túnel anatómico se reduce el estrés del injerto y aumenta la estabilidad 
anteroposterior y rotacional y por último, 3) al fijar con tornillo interferencial femoral anterógrado se logra fuerza y rigidez adecuadas para alcanzar la integración del injerto.

Como se mencionó previamente, algunos de nuestros pacientes son de nuestra práctica institucional, lo que dificulta un seguimiento a largo plazo para saber si los buenos-excelentes resultados continúan.

\section{Agradecimientos}

Al Dr. Pedro Guillen en España quien me enseñó esta técnica quirúrgica y a FEMECOT, ya que permite publicar mi cartel ganador en el Congreso Hermosillo 2019.

\section{Referencias}

1. Paschos NK, Howell SM. Anterior cruciate ligament reconstruction: principles of treatment. EFORT Open Rev. 2017; 1: 398-408.

2. Pinczewski LA, Salmon L, Heath E. ACL reconstruction with hamstring tendons. In: Sccott WN, Insall \& Scott. Surgery of the knee. 6th edit. Philadelphia. Elsevier 2018, pp. 631-641.

3. Lord B, Grice J, Cox G, Yasen S, Wilson A. ACL reconstructionEvolution \& current concepts. Orthop Trauma. 2014; 29 (1): 12-23.

4. Murawski CD, van Eck CF, Irrgang JJ, Tashman S, Fu FH. Operative treatment of primary ACL rupture in adults. J Bone Joints Surg Am. 2014; 96 (8): 685-694.
5. Guillen I, Guillen P, Garcia SO, Ordonez S, Maestro A, Rodriguez $\mathrm{L}$, et al. Innervation of partially inserted human tendons used to reconstruct ACL. MOJ Anat \& Physiol 2017; 3 (2): 00085.

6. Gupta R, Bahadur R, Malhotra A, Masih GD, Gupta P. Anterior cruciate ligament reconstruction using Hamstring tendon autograft with preserved insertion. Arthroscopy Tech. 2016; 5 (2): e269-e274.

7. Shino K, Suzuki T, Iwahashi T, Mae T, Nakamura N, Nakata K, Nakagawa S. The resident's ridge as an arthroscopic landmark for anatomical femoral tunnel drilling in ACL reconstruciton. Knee Surg Sports Traumatol Arhtrosc 2010; 18: 1164-1168.

8. Lubowitz JH, Akhavan S, Waterman BR, Aalami-Harandi A, Konicek J. Technique for creating to ACL femoral socket: Optimizing femoral footprint anatomic restoration using Outside-In drilling. Arthroscopy. 2013; 29 (3): 522-528.

9. Nishimoto K, Kuroda R, Mizuno K, Hoshino Y, Nagamune K, Kubo S, et al. Analysis of the Graft bending angle at the femoral tunnel aperture in anatomic double bundle ACL rerconstruction: a comparison of the transtibial \& the far anteromedial portal technique. Knee Surg Sports Traumatol Arhtrosc. 2009; 17: 270-276.

10. Brand J Jr, Weiler A, Caborn DN, Brown CH Jr, Johnson DL. Graft fixation in cruciate ligament reconstruction. Am J Sports Med. 2000; 28 (5): 761-774.

11. Whitehead TS. Failure of ACL reconstruction. Clin Sports Med. 2013; 32: 177-204

12. Malempati CS, Metzler AV, Johnson DL. Single-bundle anatomic ACL reconstruction. Surgical Technique Pearls \& Pitfallls. Clin Sports Med. 2017; 36 (1): 53-70.

\section{Conflicto de intereses}

Los autores declaran no tener ningún conflicto de intereses en la publicación de este artículo. 